\title{
1 Benchmarking predictions of MHC class I restricted T cell epitopes
}

3 Short title: $\mathrm{MHC}$ I epitope prediction benchmarking

4

5 Sinu Paul ${ }^{1}$, Nathan P. Croft ${ }^{2,3}$, Anthony W. Purcell ${ }^{2,3}$, David C. Tscharke ${ }^{4}$, Alessandro

6 Sette $^{1,5}$ Morten Nielsen ${ }^{6,7}$ and Bjoern Peters ${ }^{1,5^{*}}$

8 1. Division of Vaccine Discovery, La Jolla Institute for Immunology, La Jolla, CA

9 2. Infection and Immunity Program, Biomedicine Discovery Institute, Monash

10 University, Clayton, VIC 3800, Australia

11 3. Department of Biochemistry and Molecular Biology, Monash University, Clayton, VIC

123800 , Australia

13 4. John Curtin School of Medical Research, The Australian National University,

14 Canberra, ACT 2601, Australia

15 5. Department of Medicine, University of California, San Diego, La Jolla, CA 92093

16 6. Department of Bio and Health Informatics, Technical University of Denmark, DK $17 \quad 2800$ Lyngby, Denmark

18 7. Instituto de Investigaciones Biotecnológicas, Universidad Nacional de San Martín, CP1650 San Martín, Argentina

20

$21{ }^{*}$ Corresponding author

22 E-mail: bpeters@lji.org 


\section{Abstract}

T cell epitope candidates are commonly identified using computational prediction

25 tools in order to enable applications such as vaccine design, cancer neoantigen

26 identification, development of diagnostics and removal of unwanted immune responses

27 against protein therapeutics. Most T cell epitope prediction tools are based on machine

28 learning algorithms trained on $\mathrm{MHC}$ binding or naturally processed $\mathrm{MHC}$ ligand elution

29 data. The ability of currently available tools to predict $\mathrm{T}$ cell epitopes has not been

30 comprehensively evaluated. In this study, we used a recently published dataset that

31 systematically defined $\mathrm{T}$ cell epitopes recognized in vaccinia virus (VACV) infected

32 mice, considering both peptides predicted to bind $\mathrm{MHC}$ or experimentally eluted from

33 infected cells, making this the most comprehensive dataset of $\mathrm{T}$ cell epitopes mapped in

34 a complex pathogen. We evaluated the performance of all currently publicly available

35 computational $\mathrm{T}$ cell epitope prediction tools to identify these major epitopes from all

36 peptides encoded in the VACV proteome. We found that all methods were able to

37 improve epitope identification above random, with the best performance achieved by

38 neural network-based predictions trained on both $\mathrm{MHC}$ binding and $\mathrm{MHC}$ ligand elution

39 data (NetMHCPan-4.0 and MHCFlurry). Impressively, these methods were able to

40 capture more than half of the major epitopes in the top $0.04 \%(\mathrm{~N}=277)$ of peptides in

41 the VACV proteome $(\mathrm{N}=767,788)$. These performance metrics provide guidance for

42 immunologists as to which prediction methods to use. In addition, this benchmark was

43 implemented in an open and easy to reproduce format, providing developers with a

44 framework for future comparisons against new tools. 


\section{Author summary}

Computational prediction tools are used to screen peptides to identify potential T cell epitope candidates. These tools, developed using machine learning methods, save

49 time and resources in many immunological studies including vaccine discovery and cancer neoantigen identification. In addition to the already existing methods several

51 epitope prediction tools are being developed these days but they lack a comprehensive

52 and uniform evaluation to see which method performs best. In this study we did a

53 comprehensive evaluation of publicly accessible $\mathrm{MHC}$ I restricted $\mathrm{T}$ cell epitope

54 prediction tools using a recently published dataset of Vaccinia virus epitopes. We found

55 that methods based on artificial neural network architecture and trained on both MHC

56 binding and ligand elution data showed very high performance (NetMHCPan-4.0 and

57 MHCFlurry). This benchmark analysis will help immunologists to choose the right

58 prediction method for their desired work and will also serve as a framework for tool

59 developers to evaluate new prediction methods.

61 1. Introduction

62

63 T cell epitope identification is important in many immunological applications including

64 development of vaccines and diagnostics in infectious, allergic and autoimmune

65 diseases, removal of unwanted immune responses against protein therapeutics and in

66 cancer immunotherapy. Computational T cell epitope prediction tools can help to reduce

67 the time and resources needed for epitope identification projects by narrowing down the

68 peptide repertoire that needs to be experimentally tested. Most epitope prediction tools 
69 are developed using machine learning algorithms trained on two types of experimental

70 data: binding affinities of peptides to specific $\mathrm{MHC}$ molecules generated using $\mathrm{MHC}$

71 binding assays, or sets of naturally processed $\mathrm{MHC}$ ligands found by eluting peptides

72 from MHC molecules on the cell surface and identifying them by mass spectrometry.

73 Since the first computational epitope prediction methods were introduced more than two

74 decades ago [1-3], advancement in machine learning methods and increases in the

75 availability of training data have improved the performance of these methods

76 significantly in recent years, as has been demonstrated on benchmarks of $\mathrm{MHC}$ binding

77 data $[4,5]$.

79 Given the wealth of epitope prediction methods available, it is necessary to keep

80 comparing the performance of the different methods against each other, in order to

81 allow users to rationally decide which methods to choose, and to allow developers to

82 understand what changes can truly improve prediction performance. One issue with the

83 past evaluations has been that, when new methods are developed and tested, they are

84 commonly evaluated using the same kind of data on which they were trained, which can

85 impact the performance results. For example, a method trained using MHC binding data

86 will tend to show better performance when it is evaluated using MHC binding data and a

87 method trained using $\mathrm{MHC}$ ligand elution data will tend to perform better when

88 evaluated using $\mathrm{MHC}$ ligand data. The ultimate aim of the epitope prediction methods is

89 to predict actual $\mathrm{T}$ cell epitopes i.e. peptides that are recognized by $\mathrm{T}$ cells in the host.

90 Thus, we believe that the best way to compare prediction methods trained on different

91 data is to evaluate their performance in identifying epitopes. 
93 One problem when using T cell epitope identification as a way to benchmark prediction

94 methods is that it is typically not known what a true negative is, as only a subset of

95 epitope candidates is commonly tested for T cell recognition experimentally. Here, we

96 took advantage of a recent study that comprehensively identified $\mathrm{T}$ cell responses in

97 C57BL/6 mice infected with Vaccinia virus (VACV) [6]. This dataset is unique in that it

98 covered all peptides previously shown to be presented by either $\mathrm{H}-2 \mathrm{D}^{\mathrm{b}}$ or $\mathrm{H}-2 \mathrm{~K}^{\mathrm{b}}$

99 molecules expressed in these mice, which included epitopes identified following a large-

100 scale screen of predicted peptide ligands [7], as well as all epitopes recognized in a

101 comprehensive screen of a VACV protein expression library [8], and all peptides found

102 to be naturally processed and presented by MHC ligand elution assays using mass

103 spectrometry [6]. All these epitope candidates were rescreened in a consistent format,

104 using eight separately infected mice, defining the major epitopes (categorized as those

105 recognized in more than half of the animals), as well as negatives (never recognized in

106 any animal), and for each epitope defining the magnitude of the T cell response.

108 We retrieved predictions from all publicly available computational algorithms prior to

109 release of the dataset. We next evaluated each prediction algorithm based on its ability

110 to pick the major epitopes from within the total peptides that can be derived from VACV,

111 using different metrics such as AUC (area under the ROC curve), number of peptides

112 needed to capture different fractions of the epitopes, number of epitopes captured in the

113 top set of predicted peptides, and the magnitude of $\mathrm{T}$ cell response accounted for at 114 different thresholds. 


\section{Materials \& Methods}

\subsection{Selection of methods}

As a first step, we compiled a list of all freely available CD8+ T cell epitope prediction that had executable algorithms freely available publicly (excluding those that required us to train a prediction model), and excluding commercial prediction tools that required us

124 to obtain licenses. Out of these 44 methods, we selected those that had trained models 125 available for the two mouse alleles for which we had benchmarking data $\left(H-2 D^{b}\right.$ \& $H-$ $\left.2 \mathrm{~K}^{\mathrm{b}}\right)$. Further, we contacted the authors of the selected methods and excluded the ones

127 that the authors explicitly wanted to be excluded from the benchmarking for different reasons (mostly because the methods were not updated recently or new version of the methods were to be released soon). The final list included 15 methods that were

130 selected to be included in the benchmarking: ARB [9], BIMAS [2], IEDB Consensus [7],

131 MHCflurry [10], MHCLovac [11], NetMHC-4.0 [12], NetMHCpan-3.0 [13], NetMHCpan1324.0 [14], PAComplex [15], PREDEP [16], ProPred1 [17], Rankpep [18], SMM [19], 133 SMMPMBEC [20], SYFPEITHI [3]. Out of the 15 methods, NetMHCpan-4.0 offered two 134 different outputs, the first one being the predicted binding affinity of a peptide (referred 135 as NetMHCpan-4.0-B), and the second the predicted probability of a peptide being a 136 ligand in terms of a probability score (NetMHCpan-4.0-L). Both these outputs were 137 evaluated separately. Similarly, MHCflurry could use two different models, first one 
138 trained with only binding data (MHCflurry-B) and second one incorporating data on

139 peptides identified by mass-spectrometry (MHCflurry-L). Both these models were

140 evaluated separately. Considering these as separate methods, a total of 17 methods

141 were included in the benchmark, and are described in more detail in S1 Table. The

142 methods differed widely in the peptide lengths that they could predict for each allele. For

143 example, while MHCLovac could predict lengths 7-13 for both alleles, PAComplex could

144 predict for only 8-mers of $\mathrm{H}-2 \mathrm{~K}^{\mathrm{b}}$ and none of the lengths in case of $\mathrm{H}-2 \mathrm{D}^{\mathrm{b}}$. The methods

145 also differed in the kind of prediction scores provided but ultimately they all represented

146 a score that was intended to correlate with the probability of a peptide being an epitope

147 in the context of the given MHC molecule. A complete list of the peptide lengths allowed

148 for prediction per allele by each method and the kind of prediction scores they provide

149 are given in S2 Table.

\subsection{Dataset of VACV peptides}

153 For the benchmark analysis, we used the peptide data set described in Croft et al., 2019

154 (S3 Table). This dataset represented a comprehensive set of peptides naturally

155 processed and eluted from VACV-infected cells in addition to any previously identified

156 epitopes. The total of 220 VACV peptides were tested for $\mathrm{T}$ cell immune responses in

157 infected mice. Of these peptides, 172 were eluted from $\mathrm{H}-2 \mathrm{D}^{\mathrm{b}}$ and $\mathrm{K}^{\mathrm{b}}$ molecules from

158 VACV-infected cells as described in detail in Croft et al., 2019. In brief, DC2.4 cells

159 (derived from C57BL/6 mice [21] that expressed $\mathrm{H}-2^{\mathrm{b}} \mathrm{MHC}$ molecules were infected

160 with VACV. The $\mathrm{H}-2 \mathrm{D}^{\mathrm{b}}$ and $\mathrm{K}^{\mathrm{b}}$ molecules were then individually isolated and the bound 
161 peptides eluted. The peptides were then analyzed by high resolution liquid

162 chromatography-tandem mass spectrometry (LC-MS/MS). The remaining peptides in

163 the set were not detected by LC-MS/MS and included 46 VACV-derived $\mathrm{H}-2^{\mathrm{b}}$ restricted

164 peptides/epitopes from the IEDB [22] and one entirely unpublished epitope and another

165 that was mapped from a longer published sequence [23] identified by the Tscharke

166 laboratory. Immune reactivity for each of these 220 peptides was then tested 8 times

167 and the peptides that tested positive more than four times were classified as "major

168 epitopes" and those tested positive four or fewer times were classified as "minor

169 epitopes". All peptides that were never positive were classified as "nonimmunogenic".

170 There were 83 peptides classified as "major" positives (S3 Table), ranging in lengths 7-

171 13. In addition to the 220 peptides tested for immunogenicity, we generated all possible

172 peptides of lengths 7-13 from the VACV reference proteome

173 (https://www.uniprot.org/proteomes/UP000000344) (S4 File), which were also

174 considered non-immunogenic, based on them not being found in elution assays on

175 infected cells, and not being found positive in any of the many studies recorded in the

176 IEDB. The entire dataset comprised 767,788 peptide/allele combinations.

$178 \quad 2.3$ Performance evaluations

180 The performance of the prediction methods was evaluated mainly by generating ROC 181 curves (Receiver operating characteristic curve) and calculating the $\mathrm{AUC}_{\mathrm{ROC}}$ (Area 182 under the curve of ROC curve). The ROC curve shows the performance of a prediction 183 model by plotting the True positive rate (TPR, fraction of true positives out of the all real 
184 positives) against the False positive rate (FPR, fraction of false positives out of the all

185 real negatives) as the threshold of the predicted score is varied. $A U C_{R O C}$ is the area

186 under the ROC curve which summarizes the curve information and acts as a single

187 value representing the performance of the classifier system. A predictor whose

188 prediction is equivalent to random will have an $\mathrm{AUC}=0.5$ whereas a perfect predictor

189 will have $A \cup C=1.0$. That is, the closer the $A \cup C$ is to 1.0 , the better the prediction

190 method. AUC values were first calculated on different sets of peptides grouped by

191 length and allele separately. Secondly, overall AUCs were calculated by taking peptides

192 of all lengths and both alleles together, which reflects the real life usability of having to

193 decide which peptides to test. In this calculation, poor scores were assigned to peptides

194 of lengths where predictions were not available for a given method. For example, in the

195 case of SMM, lower numerical values of the prediction score indicate better epitope

196 candidates, with scores ranging from 0 to 100. So a score of 999 was assigned to all

197 peptides of lengths for which predictions were not available in SMM (lengths 7, 12 and

19813 for both alleles). Similarly a score of -100 was assigned in case of SYFPEITHI (H-

199 2D $:$ 7-8, 11-13; H-2K $:$ 7, 9-13) where a higher numerical value of predicted score

200 indicates better epitope candidate and the scores ranging from -4 to 32 .

201

$202 \quad$ 2.4 Fully automated pipeline to generate benchmarking metrics

203

204 The Python scikit-learn package [24] was used for calculating the AUCs and Python

205 matplotlib package [25] was used for plotting. A python script that can generate all

206 results and plots along with the input file containing all peptides and their prediction 
207 scores from each method, immunogenicity category, T cell response scores, the

208 "ProteinPilot confidence scores" representing the mass-spectrometry (MS) identification

209 confidence level of the peptides and the number of times the peptides were identified by

210 MS are provided in the GitLab repository (https://gitlab.com/iedb-tools/cd8-t-cell-

211 epitope-prediction-benchmarking). The repository also contains the outputs from the

212 script, i.e. the relevant results and plots. This will enable interested users to check our

213 results and add their own prediction algorithms.

214

$215 \quad$ 3. Results

$216 \quad 3.1$ Performance of the methods based on $\mathrm{AUC}_{\mathrm{ROC}}$

218 As described in the method section, we identified 17 distinct prediction approaches that

219 were freely accessible and could be applied to our dataset. Predictions from these 220 methods were retrieved for all peptides of lengths 7-13 in the VACV proteome, which

221 included the peptides tested for T cell response in Croft et al. (2019) [6]. The predictions 222 were done using default parameters and the prediction outputs were used as provided 223 by the tools without any modification or optimization. For tools provided by DTU server 224 (NetMHCpan, NetMHC) and IEDB (Consensus, SMM, SMMPMBEC, ARB), where it 225 provides raw score (for example predicted absolute binding affinity) and the percentile 226 ranks (predicted relative binding affinity), the percentile ranks were used in the analysis.

227 We considered the "major epitopes" (peptides that were tested positive in more than 228 four out of the eight mice) as positives. To avoid ambiguity we excluded the "minor 229 epitopes" (peptides that were tested positive in four or less mice out of the eight), and all 
230 other peptides were considered as negatives. This provides a binary classification of

231 peptides into epitopes/non-epitopes. In order to evaluate the performance of each

232 prediction approach, we generated $\mathrm{ROC}$ curves and calculated the $\mathrm{AUC}_{\mathrm{ROC}}$ for all

233 methods on a per allele $\left(H-2 D^{b}, H-2 K^{b}\right)$ and per peptide length $(7-13)$ basis, which are

234 listed in Table 1. The per allele/length AUCs were then averaged to get an AUC value

235 per each allele for each method and then the AUCs of both alleles were averaged to get

236 a single AUC value per method. These average AUC values for each method are also

237 provided in Table 1. The average AUCs varied from 0.793 to 0.983. NetMHCpan-4.0-B

238 came top based on this analysis with an average AUC of 0.983 . It was followed by

239 NetMHCpan-3.0 (AUC = 0.982) and NetMHC-4.0 (AUC =0.980). The lowest AUC was

240 obtained for MHCLovac (0.793). When looking at the individual AUC values for each

241 length, it was noticed that MHCLovac had very low performance for $\mathrm{H}-2 \mathrm{~K}^{\mathrm{b}}$ lengths 7 and

24212 (AUC of 0.529 and 0.284 respectively) where there were only one positive each.

243 Thus, these two low AUCs brought the average AUC down for MHCLovac, which is

244 arguably irrelevant, as there are very few peptides positive for those lengths in the first

245 place.

247 In practical applications, an experimental investigator uses predictions to choose which 248 peptides to synthesize and test. The total number of peptides to be synthesized and 249 tested is the limiting factor, and how many of the epitopes are covered is a measure of 250 success, regardless of what the peptide length is or what allele they are restricted by.

251 To reflect this, we estimated overall AUC values for each method by considering 252 peptides of all lengths and both alleles together. If a given prediction method was 
253 unable to make predictions for a certain length (reflecting that the length is not

254 considered likely to be an epitope), uniformly poor scores were assigned to those

255 peptides. The overall AUCs ranged from 0.642 to 0.977 . NetMHCpan-4.0-L ranked first

256 with with AUC of 0.977 followed by NetMHCpan-4.0-B (0.975) and MHCflurry-L (0.973)

257 (Table 1, Fig 1A). The ROC curves are shown in Fig 2. Fig 2A shows the ROC curves of

258 all benchmarked methods for $100 \%$ FPR and Fig 2B shows the same up to $2 \%$ FPR to

259 clearly distinguish the curves for each method in the initial part. Fig $2 \mathrm{C}$ and $2 \mathrm{D}$ show

260 respectively the same for a set of top and historically important methods. It has to be

261 noted that certain methods such as NetMHCpan-4.0 are implicitly adjusting prediction

262 scores to account for the fact that certain peptide sizes are preferred when natural

263 ligands are considered, as these methods were trained on such ligands. This means

264 that prior approaches to adjust for the prevalence of different peptide lengths as was

265 done for NetMHCPan 2.8 [26] are no longer necessary for such modern methods. It is 266 likely that other methods, such as BIMAS or SMM that were trained on binding data

267 only, could be improved when adjusting for lengths, but we wanted to test the 268 performance of each method on an as-is basis.

270 Fig 1. Bar charts showing the overall AUCs for each benchmarked method.

271 Fig 1A. Bar chart showing the overall AUCs for each method with a binary classification

272 (epitope/non-epitope) based analysis

273

274 Fig 2. ROC curves showing the performance of the benchmarked methods. The

275 curves are made by plotting true positive rate against the false positive rate in case of 
276 binary classification (epitopes/non-epitopes) based analysis and by plotting the $\%$ of $T$

277 cell response against \% of total peptides in case of T cell response based analysis.

278 Fig 2A. ROC curve for all methods that were benchmarked.

279 Fig 2B. ROC curve for all methods that were benchmarked with the curves zoomed in to

$280 \mathrm{FPR}=0.02$ in order to be able to distinguish them more clearly in this region.

281 Fig 2C. ROC curve showing the performance of a set of top and historically important 282 methods.

283 Fig 2D. ROC curve for selected methods with the curves zoomed in to FPR $=0.02$. 


\begin{tabular}{|c|c|c|c|c|c|c|c|c|c|c|c|c|c|c|c|c|c|c|c|c|c|}
\hline \multirow{3}{*}{ Method } & \multicolumn{19}{|c|}{ Binary classification (epitope/non-epitope) based } & \multicolumn{2}{|c|}{$\begin{array}{c}\mathrm{T} \text { cell response } \\
\text { based }\end{array}$} \\
\hline & \multicolumn{8}{|c|}{ H2-Db } & \multicolumn{8}{|c|}{$\mathrm{H} 2-\mathrm{Kb}$} & \multirow{2}{*}{$\begin{array}{c}\text { Averag } \\
\text { e of } \\
\text { length } \\
\text { wise } \\
\text { AUCs } \\
\text { for } \\
\text { both } \\
\text { alleles }\end{array}$} & \multirow{2}{*}{$\begin{array}{c}\text { Overall } \\
\text { AUC } \\
\text { with all } \\
\text { lengths } \\
\text { and } \\
\text { both } \\
\text { alleles } \\
\text { togethe } \\
\text { r }\end{array}$} & \multirow[b]{2}{*}{ Rank } & \multirow[b]{2}{*}{$\begin{array}{l}\text { Overall } \\
\text { AUC } \\
\text { with all } \\
\text { lengths } \\
\text { and both } \\
\text { alleles } \\
\text { together }\end{array}$} & \multirow[b]{2}{*}{ Rank } \\
\hline & 7 & 8 & 9 & 10 & 11 & 12 & 13 & $\begin{array}{c}\text { Averag } \\
\text { e of } \\
\text { length } \\
\text { wise } \\
\text { AUCs }\end{array}$ & 7 & 8 & 9 & 10 & 11 & 12 & 13 & $\begin{array}{l}\text { Avera } \\
\text { ge of } \\
\text { lengt } \\
\text { hwise } \\
\text { AUCs }\end{array}$ & & & & & \\
\hline NetMHCpan-4.0-L* & - & 0.923 & 0.986 & 0.884 & 0.997 & 1.000 & 1.000 & 0.965 & - & 0.990 & 0.988 & 0.999 & - & 1.000 & - & 0.994 & 0.979 & 0.977 & 1 & 0.979 & 1 \\
\hline NetMHCpan-4.0-B* & - & 0.943 & 0.990 & 0.912 & 0.994 & 1.000 & 1.000 & 0.973 & - & 0.989 & 0.989 & 0.996 & - & 0.999 & - & 0.993 & 0.983 & 0.975 & 2 & 0.978 & 2 \\
\hline MHCflurry-L** & - & 0.897 & 0.984 & 0.902 & 0.986 & 0.997 & 1.000 & 0.961 & - & 0.995 & 0.989 & 0.985 & - & 1.000 & - & 0.992 & 0.976 & 0.973 & 3 & 0.977 & 3 \\
\hline MHCflurry-B $B^{* *}$ & - & 0.923 & 0.983 & 0.897 & 0.981 & 0.998 & 0.999 & 0.964 & - & 0.994 & 0.988 & 0.990 & - & 0.999 & - & 0.993 & 0.978 & 0.972 & 4 & 0.976 & 4 \\
\hline NetMHCpan-3.0 & - & 0.955 & 0.989 & 0.900 & 0.996 & 0.999 & 0.999 & 0.973 & - & 0.988 & 0.986 & 0.996 & - & 0.999 & - & 0.992 & 0.982 & 0.972 & 5 & 0.975 & 5 \\
\hline NetMHC-4.0 & - & 0.945 & 0.990 & 0.902 & 0.995 & 1.000 & 0.998 & 0.972 & - & 0.989 & 0.981 & 0.990 & - & 0.994 & - & 0.989 & 0.980 & 0.969 & 6 & 0.974 & 6 \\
\hline IEDB Consensus & - & 0.813 & 0.991 & 0.879 & 0.870 & 1.000 & 0.998 & 0.925 & - & 0.988 & 0.977 & 0.993 & - & 0.994 & - & 0.988 & 0.957 & 0.960 & 7 & 0.961 & 7 \\
\hline SMMPMBEC & - & 0.498 & 0.988 & 0.924 & 0.733 & - & - & 0.786 & - & 0.986 & 0.971 & 0.977 & - & - & 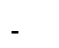 & 0.978 & 0.882 & 0.938 & 8 & 0.940 & 8 \\
\hline SMM & & 0.490 & 0.989 & 0.864 & 0.687 & - & - & 0.757 & - & 0.984 & 0.969 & 0.979 & - & - & - & 0.977 & 0.867 & 0.935 & 9 & 0.938 & 10 \\
\hline ARB & - & 0.623 & 0.988 & 0.862 & 0.916 & - & - & 0.847 & - & 0.978 & 0.981 & 0.927 & - & - & - & 0.962 & 0.905 & 0.928 & 10 & 0.939 & 9 \\
\hline Rankpep & - & 0.629 & 0.991 & 0.923 & 0.908 & - & - & 0.863 & - & 0.986 & 0.819 & - & - & - & - & 0.903 & 0.883 & 0.927 & 11 & 0.894 & 12 \\
\hline BIMAS & - & - & 0.981 & 0.886 & - & - & - & 0.933 & - & 0.968 & 0.868 & 0.990 & - & - & - & 0.942 & 0.938 & 0.909 & 12 & 0.918 & 11 \\
\hline MHCLovac & - & 0.887 & 0.949 & 0.942 & 0.987 & 0.987 & 0.993 & 0.957 & 0.529 & 0.876 & 0.723 & 0.728 & - & 0.284 & - & 0.628 & 0.793 & 0.878 & 13 & 0.863 & 13 \\
\hline SYFPEITHI & - & - & 0.988 & 0.891 & - & - & - & 0.939 & - & 0.983 & - & - & - & - & - & 0.983 & 0.961 & 0.813 & 14 & 0.778 & 14 \\
\hline PREDEP & - & - & 0.781 & - & - & - & - & 0.781 & - & 0.844 & - & - & - & - & - & 0.844 & 0.813 & 0.770 & 15 & 0.737 & 15 \\
\hline ProPred-I & - & - & 0.981 & - & - & - & - & 0.981 & - & - & 0.869 & - & - & - & - & 0.869 & 0.925 & 0.687 & 16 & 0.651 & 17 \\
\hline PAComplex & - & - & - & - & - & - & - & - & - & 0.902 & - & - & - & - & - & 0.902 & 0.902 & 0.642 & 17 & 0.652 & 16 \\
\hline
\end{tabular}

286

287 The table shows the AUCs for each method on a per allele/length basis where allele/lengths are available and the average AUCs

288 per method per alleles derived from the lengthwise AUCs. The overall AUCs show the AUCs calculated with all lengths and both 
289 alleles taken together for each method and these values are used to rank the performance of the methods. Additionally, the AUCs

290 derived based on the T cell response obtained for each peptide/allele combination are also shown.

291 *NetMHCpan-4.0: B - using binding based prediction; L - using ligand based prediction

$292{ }^{* *}$ MHCflurry: B - models trained on binding affinity measurements; L - Mass-spec datasets incorporated 


\subsection{Alternative metrics to evaluate performance of the methods}

In addition to the AUCs, we calculated metrics that are more intuitive for scientists less

296 familiar with ROC curves, namely the number of peptides needed to capture $50 \%, 75 \%$

297 and $90 \%$ of the epitopes (which corresponds to comparing ROC curves at horizontal

298 lines at $50 \%, 75 \%$ and $90 \%$ sensitivity). Since a total of 83 major epitopes were found in 299 the dataset, we calculated how many predicted peptides were needed to capture 42 (= $30050 \%$ ) of them, after sorting based on the prediction score for each method. The results 301 are shown in Table 2 and Fig 3A. The number of peptides required by the methods 302 varied widely. NetMHCpan-4.0-L required only $0.036 \%(\mathrm{~N}=277)$ peptides and 303 MHCflurry-L needed only $0.037 \%(\mathrm{~N}=285)$ peptides to capture $50 \%$ epitopes while 304 ProPred1 needed 21\% $(160,644)$ and PAComplex needed 30\% $(230,132)$ peptides 305 respectively to capture $50 \%$ epitopes. In a similar manner, we also calculated the 306 number of peptides needed to capture $75 \%(\mathrm{~N}=62)$ and $90 \%$ epitopes $(\mathrm{N}=75)$. For $30775 \%$ epitopes, MHCflurry-B was on top with $0.20 \%$ peptides $(N=1,542)$ whereas 308 PAComplex needed 65\% peptides ( $\mathrm{N}=498,917$ ) (Table 2, Fig 3B). For 90\% epitopes 309 NetMHCpan-4.0-B needed only $1.33 \%(\mathrm{~N}=10,224)$ peptides and NetMHCpan-4.0-L 310 required only $1.47 \%(11,254)$ peptides while ProPred 1 and PAComplex needed $84 \%(\mathrm{~N}$ $311=646,291)$ and $86 \%(660,189)$ peptides respectively (Table 2, Fig 3C).

313 Similar to above, another metric we calculated was the number of epitopes captured in

314 the top 172 peptides predicted by each method. This corresponds to the number of 315 peptides identified by mass-spectrometry of naturally eluted ligands. The results are 
316 provided in Table 3 and Fig 4A. The number of epitopes captured by these top peptides

317 also varied widely for the methods. The MHCflurry methods performed the best,

318 capturing $43 \%(\mathrm{~N}=36)$ of the epitopes and NetMHCpan-4.0 methods captured $40 \%(\mathrm{~N}$

$319=33$ ) epitopes while PREDEP could not capture any epitope in the top 172 peptides.

321 In addition to the analyses based on the binary classification of peptides (epitopes/non322 epitopes), we also evaluated the methods based on the $\mathrm{T}$ cell response generated by 323 the peptides, measured as the percentage of IFNy producing cells in CD8 T cells as a 324 whole (S3 Table). First, we plotted the cumulative fraction of the $\mathrm{T}$ cell response 325 accounted for by a given \% of the total peptides considered and estimated the overall 326 AUCs for each method with peptides of all lengths and both alleles taken together. 327 Measuring the performance of the prediction methods based on the magnitude of the $\mathrm{T}$ 328 cell response covered systematically gave slightly higher performances with overall 329 AUCs ranging from 0.651 to 0.979 (Table 1, Fig 1B). The rankings however were 330 essentially identical, with NetMHCpan-4.0-L again ranking first with an AUC of 0.979 331 followed by NetMHCpan-4.0-B (0.978) and MHCflurry-L of (0.977). Fig 2E shows the 332 the corresponding curves for $100 \%$ peptides and Fig $2 \mathrm{~F}$ shows the same for $2 \%$ 333 peptides. Similar to the analysis we did with epitopes, we also estimated the number of 334 peptides needed to capture $50 \%, 75 \%$ and $90 \%$ of the $\mathrm{T}$ cell response. The results 335 were essentially same as that of the epitopes at the corresponding percentage levels 336 with some minor exceptions (Table 2, Fig 3D-F). Similarly we also calculated the 337 amount of $\mathrm{T}$ cell response captured in the top 172 peptides predicted by each method 
338 (Table 3 and Fig 4B). Here NetMHCpan-4.0-B came top with $47.4 \%$ of the response

339 and was closely followed by MHCflurry-B with $47.2 \% \mathrm{~T}$ cell response.

341 Fig 1. Bar charts showing the overall AUCs for each benchmarked method.

342 Fig 1B. Bar chart showing the overall AUCs for each method with a $\mathrm{T}$ cell response

343 based analysis

Fig 2. ROC curves showing the performance of the benchmarked methods. The

347 curves are made by plotting true positive rate against the false positive rate in case of

348 binary classification of peptides (epitopes/non-epitopes) based analysis and by plotting

349 the $\%$ of $\mathrm{T}$ cell response against \% of total peptides in case of $\mathrm{T}$ cell response based 350 analysis.

351 Fig 2E. Curve generated by plotting the \% of $\mathrm{T}$ cell response against $\%$ of total 352 peptides.

353 Fig 2F. Curve generated by plotting the \% of T cell response against \% of total peptides.

354 This plot shows the curves zoomed in to $\%$ of peptides $=0.02$.

Fig 3. Number of peptides needed to capture $50 \%, 75 \%$ asnd $90 \%$ epitopes and T

357 cell response

358 Fig 3 A. Number of peptides needed to capture $50 \%$ epitopes.

359 Fig 3B. Number of peptides needed to capture $75 \%$ epitopes.

360 Fog 3C. Number of peptides needed to capture $90 \%$ epitopes. 
361 Fig 3D. Number of peptides needed to capture 50\% T cell response.

362 Fog 3E. Number of peptides needed to capture $75 \%$ T cell response.

363 Fog 3F. Number of peptides needed to capture 90\% T cell response.

365 Fig 4. Number of epitopes and the amount of $\mathrm{T}$ cell response captured in the top

366172 peptides. The number of top peptides was fixed at 172 to match the number of 367 peptides identified by mass-spectrometry.

368 Fig 4A. Number of epitopes captured in the top 172 peptides.

369 Fig 4B. Amount of T cell response captured in the top 172 peptides.

371 Table 2. Number of peptides needed to capture $50 \%, 75 \%$ and $90 \%$ of epitopes

372 and T cell response 


\begin{tabular}{|c|c|c|c|c|c|c|c|c|c|c|c|c|c|c|}
\hline \multirow{3}{*}{ Method } & \multicolumn{4}{|c|}{ Peptides needed to capture $50 \%$} & \multirow{3}{*}{ Method } & \multicolumn{4}{|c|}{ Peptides needed to capture $75 \%$} & \multirow{3}{*}{ Method } & \multicolumn{4}{|c|}{ Peptides needed to capture $90 \%$} \\
\hline & \multicolumn{2}{|c|}{ Epitopes } & \multicolumn{2}{|c|}{ T cell response } & & \multicolumn{2}{|c|}{ Epitopes } & \multicolumn{2}{|c|}{ T cell response } & & \multicolumn{2}{|c|}{ Epitopes } & \multicolumn{2}{|c|}{ T cell response } \\
\hline & Count & $\%$ & Count & $\%$ & & Count & $\%$ & Count & $\%$ & & Count & $\%$ & Count & $\%$ \\
\hline NetMHCpan-4.0-L & 277 & $0.04 \%$ & 286 & $0.04 \%$ & MHCflurry-B & 1,542 & $0.20 \%$ & 1,639 & $0.21 \%$ & NetMHCpan-4.0-B & 10,224 & $1.33 \%$ & 8,030 & $1.05 \%$ \\
\hline MHCflurry-L & 285 & $0.04 \%$ & 230 & $0.03 \%$ & MHCflurry-L & 1,896 & $0.25 \%$ & 1,991 & $0.26 \%$ & NetMHCpan-4.0-L & 11,254 & $1.47 \%$ & 11,309 & $1.47 \%$ \\
\hline MHCflurry-B & 307 & $0.04 \%$ & 216 & $0.03 \%$ & NetMHCpan-4.0-L & 2,147 & $0.28 \%$ & 1,549 & $0.20 \%$ & MHCflurry-B & 13,719 & $1.79 \%$ & 13,842 & $1.80 \%$ \\
\hline NetMHCpan-4.0-B & 349 & $0.05 \%$ & 236 & $0.03 \%$ & NetMHCpan-4.0-B & 3,058 & $0.40 \%$ & 2,250 & $0.29 \%$ & MHCflurry-L & 15,651 & $2.04 \%$ & 16,039 & $2.09 \%$ \\
\hline NetMHC-4.0 & 365 & $0.05 \%$ & 317 & $0.04 \%$ & NetMHC-4.0 & 3,922 & $0.51 \%$ & 3,037 & $0.40 \%$ & NetMHCpan-3.0 & 27,731 & $3.61 \%$ & 17,533 & $2.28 \%$ \\
\hline SMM & 924 & $0.12 \%$ & 761 & $0.10 \%$ & IEDB Consensus & 4,925 & $0.64 \%$ & 4,877 & $0.64 \%$ & NetMHC-4.0 & 30,472 & $3.97 \%$ & 20,984 & $2.73 \%$ \\
\hline IEDB Consensus & 1,163 & $0.15 \%$ & 1,135 & $0.15 \%$ & NetMHCpan-3.0 & 5,764 & $0.75 \%$ & 5,341 & $0.70 \%$ & IEDB Consensus & 49,777 & $6.48 \%$ & 44,516 & $5.80 \%$ \\
\hline Rankpep & 1,251 & $0.16 \%$ & 3,211 & $0.42 \%$ & SMM & 6,240 & $0.81 \%$ & 5,493 & $0.72 \%$ & SMMPMBEC & 71,593 & $9.33 \%$ & 91,619 & $11.93 \%$ \\
\hline NetMHCpan-3.0 & 1,309 & $0.17 \%$ & 1,157 & $0.15 \%$ & SMMPMBEC & 7,939 & $1.03 \%$ & 7,174 & $0.93 \%$ & SMM & 83,425 & $10.87 \%$ & 84,821 & $11.05 \%$ \\
\hline SMMPMBEC & 1,697 & $0.22 \%$ & 1,214 & $0.16 \%$ & Rankpep & 16,218 & $2.11 \%$ & 34,742 & $4.53 \%$ & Rankpep & 131,992 & $17.19 \%$ & 399,634 & $52.05 \%$ \\
\hline ARB & 1,781 & $0.23 \%$ & 2,262 & $0.29 \%$ & ARB & 17,260 & $2.25 \%$ & 13,791 & $1.80 \%$ & ARB & 152,456 & $19.86 \%$ & 91,256 & $11.89 \%$ \\
\hline SYFPEITHI & 2,070 & $0.27 \%$ & 1,955 & $0.25 \%$ & BIMAS & 20,156 & $2.63 \%$ & 17,264 & $2.25 \%$ & MHCLovac & 285,408 & $37.18 \%$ & 312,869 & $40.75 \%$ \\
\hline BIMAS & 4,466 & $0.58 \%$ & 6,733 & $0.88 \%$ & MHCLovac & 138,245 & $18.01 \%$ & 187,337 & $24.40 \%$ & BIMAS & 313,329 & $40.81 \%$ & 166,819 & $21.73 \%$ \\
\hline PREDEP & 30,363 & $3.96 \%$ & 31,820 & $4.14 \%$ & SYFPEITHI & 267,557 & $34.85 \%$ & 351,034 & $45.72 \%$ & SYFPEITHI & 567,644 & $73.94 \%$ & 601,086 & $78.29 \%$ \\
\hline MHCLovac & 34,218 & $4.46 \%$ & 30,981 & $4.04 \%$ & PREDEP & 327,655 & $42.68 \%$ & 388,964 & $50.66 \%$ & PREDEP & 591,684 & $77.07 \%$ & 616,259 & $80.26 \%$ \\
\hline ProPred1 & 160,644 & $20.93 \%$ & 221,775 & $28.89 \%$ & ProPred1 & 464,173 & $60.46 \%$ & 494,782 & $64.44 \%$ & ProPred1 & 646,291 & $84.19 \%$ & 658,585 & $85.78 \%$ \\
\hline PAComplex & 230,132 & $29.98 \%$ & 216,523 & $28.19 \%$ & PAComplex & 498,917 & $64.99 \%$ & 492,155 & $64.10 \%$ & PAComplex & 660,189 & $86.00 \%$ & 657,535 & $85.64 \%$ \\
\hline
\end{tabular}

374 The table shows the number of peptides needed to capture $50 \%, 75 \%$ and $90 \%$ of epitopes and T cell response. The lower the

375 number of peptides needed to capture the respective amount of epitopes or T cell response, the better the performance of the

376 prediction method. 
377 Table 3. Number of epitopes and amount of T cell response captured in the top 172 378 peptides.

\begin{tabular}{|c|c|c|c|c|}
\hline \multirow[t]{2}{*}{ Method } & \multicolumn{2}{|c|}{$\begin{array}{l}\text { Epitopes captured in } \\
\text { top } 172 \text { peptides }\end{array}$} & \multicolumn{2}{|c|}{$\begin{array}{c}\text { T cell response } \\
\text { captured in top } 172 \\
\text { peptides }\end{array}$} \\
\hline & Count & $\%$ & Count & $\%$ \\
\hline MHCflurry-L & 36 & $43.37 \%$ & 24.92 & $44.36 \%$ \\
\hline MHCflurry-B & 36 & $43.37 \%$ & 26.51 & $47.18 \%$ \\
\hline NetMHCpan-4.0-B & 33 & $40.00 \%$ & 26.64 & $47.42 \%$ \\
\hline NetMHCpan-4.0-L & 33 & $39.76 \%$ & 22.7 & $40.40 \%$ \\
\hline NetMHC-4.0 & 31 & $36.86 \%$ & 23.5 & $41.83 \%$ \\
\hline Rankpep & 22 & $26.51 \%$ & 11.1 & $19.75 \%$ \\
\hline SYFPEITHI & 16 & $19.73 \%$ & 9.43 & $16.78 \%$ \\
\hline ProPred1 & 13 & $15.66 \%$ & 6.28 & $11.17 \%$ \\
\hline ARB & 12 & $14.46 \%$ & 7.32 & $13.04 \%$ \\
\hline BIMAS & 11 & $13.25 \%$ & 4.64 & $8.25 \%$ \\
\hline SMM & 11 & $12.67 \%$ & 7.65 & $13.61 \%$ \\
\hline NetMHCpan-3.0 & 10 & $12.11 \%$ & 9.23 & $16.42 \%$ \\
\hline SMMPMBEC & 7 & $8.72 \%$ & 7.03 & $12.51 \%$ \\
\hline PAComplex & 3 & $3.61 \%$ & 4.1 & $7.30 \%$ \\
\hline IEDB Consensus & 2 & $2.88 \%$ & 1.93 & $3.43 \%$ \\
\hline MHCLovac & 2 & $2.41 \%$ & 1.51 & $2.69 \%$ \\
\hline PREDEP & 0 & $0.00 \%$ & 0 & $0.00 \%$ \\
\hline
\end{tabular}

380 Number of epitopes and amount of T cell response captured in the top 172 peptides.

381 The higher the number of epitopes or amount of T cell response captured, the better the 382 performance of the prediction method. The number of top peptides was fixed at 172 383 because that was the number of peptides identified by LC-MS/MS. 


\subsection{Comparing epitope identification by mass-spectrometry and epitope}

\section{prediction}

Next, we wanted to determine how epitope candidates identified experimentally by

mass-spectrometry (MS) should be ranked. In the dataset used, a single elution and

390 identification of peptides by LC-MS/MS was done. Rather than treating the outcome of 391 this MS experiment as a binary outcome (ligands being identified or not), we ranked the

392 results based on confidence that the identified hits are accurate, and to test if that 393 enables discriminating hits that turn out to be epitopes from others that do not. We 394 compared the performance of three metrics derived from the MS experiment. First the 395 ProteinPilot confidence score which is obtained from the software used in identification 396 of peptides using MS; second, the number of times a peptide was identified in MS (i.e. 397 spectral count); and third, a combined score derived by taking the product of the previous two (S3 Table). When evaluating these three approaches, we found that the number of times the peptide was identified by MS had the best performance with an

$400 \mathrm{AUC}$ of 0.674 (AUC of combined score $=0.667$, ProteinPilot $=0.503$ ). This shows that

401 the number of times a precursor ion was selected for MS/MS, which is a proxy for the 402 abundance of a peptide, but not the ProteinPilot score, which is an indication of the 403 certainty of the hit, has small but significant predictive power for a peptide to be an 404 actual epitope $(p=0.0001)$.

405

406 Using this score to rank the identified MS ligands, and assigning a score of 0 to all other 407 peptides in the VACV peptide dataset, we could now generate ROC curves in the same 
way as was done for the prediction approaches, and compare it to the best performing

409 method NetMHCpan-4.0-L. Fig 5A shows the ROC curves for both MS-based and

410 prediction based (NetMHCpan-4.0-L) approaches for 100\% FPR and Fig 5B shows the

411 ROC curves up to $2 \%$ FPR. The MS based curve had an AUC of 0.898 compared to

412 AUC of 0.977 for NetMHCpan-4.0-L. At the same time, when evaluating how many

413 peptides are needed to be synthesized to capture $50 \%$ of the epitopes, the ligand

414 elution data by far outperforms all prediction methods, needing only $0.01 \%$ peptides ( $N$

$415=48$ ), with the best prediction method (NetMHCPan4L) needing 277 peptides. This

416 suggests that, when the intent of a study is to identify all epitopes, and the number of

417 peptides tested is a minor concern, predictions have a better performance, as some

418 fraction of $\mathrm{T}$ cell epitopes will be missed in typical ligand-elution experiments. At the 419 same time, when the intent is to identify a small pool of high confidence candidate 420 peptides, MHC ligand elution experiments have a much better performance.

Fig 5. ROC curves comparing epitope candidate selection using mass-

423 spectrometry and prediction approaches. The curves were generated from the

424 number times a precursor ion was selected for MS/MS which acts as a proxy for the

425 abundance of a peptide and represents MS and NetMHCpan-4.0-L prediction scores.

426 Fig 5A. ROC curves comparing epitope candidate selection using mass-spectrometry

427 and prediction approaches. Plot showing 100\% FPR.

428 Fig 5B. ROC curves comparing epitope candidate selection using mass-spectrometry 429 and prediction approaches. Plot showing up to $2 \%$ FPR. 


\subsection{Comparison of prediction speed}

As an independent measure of prediction performance, we wanted to compare the

434 speed with which the different methods could provide their answers. As the initial gathering of predictions involved significant manual troubleshooting, we performed a

436 dedicated speed test, using 5 random amino acid sequences that were 1000 residues

437 long for both $\mathrm{H}-2 \mathrm{D}^{\mathrm{b}}$ and $\mathrm{H}-2 \mathrm{~K}^{\mathrm{b}}$ alleles, and for each method. We used the fastest

438 available online versions of the methods for prediction, for example, RESTful API where

439 available. For some methods, we were unable to quantify prediction times that could be

440 meaningfully compared to the others, and these were excluded from this analysis (for

441 example, MHCflurry server was having memory issues and we could not get the

442 predictions done in a manner consistent with other methods). Out of the 10 methods

443 that we could compare, BIMAS and SYFPEITHI were the fastest with 0.97 and 0.99

444 seconds per sequence respectively (Fig 6A). On the other end, NetMHCpan-4.0 and

445 NetMHCpan-3.0 were the slowest with average times of 8.53 and 6.30 seconds. We

446 noticed that in general, matrix based methods (BIMAS, SYFPEITHI, RANKPEP, SMM,

447 SMMPMBEC) were significantly faster compared to artificial neural network-based

448 methods (NetMHCpan-4.0, NetMHCpan-3.0, NetMHC-4.0) on average (Fig 6B). The

449 matrix-based methods took an average of 2.07 seconds while the neural network-based

450 methods needed an average of 6.06 seconds per sequence, with the pan-based

451 methods being particularly slow. This indicates a trade-off between prediction

452 performance and speed. 
454 Fig 6. Comparison of prediction speed among the some of the benchmarked

455 methods. The plot shows the average time in seconds taken by the methods for doing

456 epitope prediction for 1000 amino acid residue long sequence.

457 Fig 6 A. Comparison of prediction speed among individual methods

458 Fig 6B. Comparison of prediction speed between matrix-based methods and artificial

459 neural network-based methods

460

\section{Discussion}

463 In this study we comprehensively evaluated the ability of different prediction methods to 464 identify $\mathrm{T}$ cell epitopes. We found that most of the latest methods perform at a very high 465 level, especially the methods developed on artificial neural-network based architectures.

466 In addition, we found that methods that integrated $\mathrm{MHC}$ binding and $\mathrm{MHC}$ ligand elution 467 data performed better than those trained on MHC binding data alone. And where 468 available, methods that provided two outputs, where one output predicted MHC ligands 469 vs. another that predicted MHC binding, the MHC ligand output score performed better.

470 Based on these results, the IEDB will be updating the default recommended prediction

471 method to NetMHCPan-4.0-L.

472

473 Our results highlight the value of integrating both $\mathrm{MHC}$ binding and $\mathrm{MHC}$ elution data 474 into training prediction algorithms, and confirms that the approach of generating 475 different prediction outputs allows to capture aspects of MHC ligands that is not 476 captured by binding alone, and that these aspects improve $\mathrm{T}$ cell epitope predictions 
477 [14]. At the same time, the difference in performance is small, highlighting that $\mathrm{MHC}$ 478 binding captures nearly all features of peptides that distinguish epitopes from non479 epitopes in current prediction methods.

481 It is also interesting to note that the top 172 peptides captured $40 \%$ or more epitopes by 482 the top methods (NetMHCpan-4.0, MHCflurry) (Table 3). This should be viewed against 483 the total amount of peptides in the entire peptidome that could be generated from VACV 484 proteome. It means that the top $0.02 \%$ of the peptides could capture $40 \%$ of the 485 epitopes and close to $50 \%$ of the total immune response (Table 3). Similarly, it took less 486 than $2 \%$ of the top peptides predicted by the best methods to capture $90 \%$ of the 487 epitopes and T cell response. In the same manner less than $0.04 \%$ of peptides captured $48850 \%$ of the epitopes and $\mathrm{T}$ cell response (Table 2 ). This is relevant because it shows 489 that these methods can significantly reduce the number of peptides needed to be tested 490 in large scale epitope identification studies. Balance between greater coverage (with 491 fewer false negatives) vs. greater specificity (with fewer false positives) that comes with 492 different thresholds and methods has to be made in the context of a specific application. 493 For example, if the goal of a study is to identify patient specific tumor epitopes for a low 494 mutational burden tumor, avoiding false negatives is crucial, as there are few potential 495 targets to begin with. In contrast, if the goal of a study is to identify epitopes that can be 496 used as potential diagnostic markers for a bacterial infection, there will be a plethora of 497 candidates, and avoiding false positives becomes much more important. 
A limitation of previous benchmarks is that they either used $\mathrm{MHC}$ binding or $\mathrm{MHC}$ ligand

500 elution data to evaluate performance, or they use T cell epitope datasets for which it is

501 unclear what constitutes a negative. The dataset we use here is unique in that it 502 comprehensively defines $\mathrm{T}$ cell epitopes in a consistent fashion. The downside of this

503 dataset is that it is limited to two murine MHC class I molecules. Future benchmarks on

504 similar datasets for T cell epitopes recognized in humans will be necessary to confirm

505 that the results hold there.

507 In the process of conducting this benchmark, it became clear that comparing methods

508 that varied in terms of the lengths of peptides they covered introduces difficulties.

509 Developers want to see methods compared on the same datasets, and can refer to the

510 values in Table 1. We strongly advocate that all prediction methods should be evaluated

511 by ranking all possible peptides, which should be extended to ligands from 7 to 15

512 residues in the case of $\mathrm{MHC}$ class I. Method developers should also include guidance

513 on how scores from different length peptides should be compared. That has been done

514 in some cases before [26], but has not been done in others, including in several

515 developed by our own team (SMM, SMMPMBEC).

517 We want to mention that out of the 172 peptides that were identified by LC-MS/MS, 37

518 were detected in modified form but were tested for immunogenicity as synthesized

519 unmodified peptides (S3 Table). The caveat is that we do not know to what extent the

520 modification affects binding compared to unmodified form for these peptides or indeed if

521 some modification were artefacts of sample preparation. We therefore repeated the 
522 analysis after excluding the peptides identified in modified form and found that the

523 AUCs did not change much and the rankings of the methods remained same except

524 that MHCflurry-B moved ahead of MHCflurry-L (S5 Table).

525

526 Although the artificial neural network-based methods were much ahead in performance,

527 they were found to be slower compared to the matrix-based methods. This is expected

528 since artificial neural network-based methods employ more complex algorithms

529 compared to rather linear models used by matrix-based methods. But it should be noted

530 that offline or standalone versions are available for many methods that are significantly

531 faster than the online and API versions. These versions can be run on local computers

532 and users should consider using these standalone versions for doing large scale

533 predictions.

534

535 Finally, an important aspect of this benchmark is that we have made all data including

536 prediction results from all benchmarked methods and the code for generating all result

537 metrics and plots publicly available as a pipeline (https://gitlab.com/iedb-tools/cd8-t-cell-

538 epitope-prediction-benchmarking). We believe this will act as a useful resource for

539 streamlined benchmarking process for epitope prediction methods. New prediction

540 method developers can plug in the prediction scores from the new method into this

541 dataset and run the pipeline for side-by-side comparison of their method's performance

542 with those included in the analysis. The only point to remember is that the developers

543 should exclude this data from the training data for their method. We believe that this

544 benchmark analysis will not only help guide immunologists choose the best epitope 
545 prediction methods for their intended use, but will also help method developers evaluate

546 and compare new advances in method development, and provide target metrics to

547 optimize against.

548

\section{Author contributions}

550

551 BP and SP designed the study. SP retrieved predictions, and performed all analysis.

552 NPC, AWP and DCT aided in the interpretation of the MS data in the context of

553 predictions. All authors contributed to the interpretation of the results and writing of the

554 manuscript.

555

$556 \quad 6 . \quad$ References

557 1. Sette A, Buus S, Appella E, Smith JA, Chesnut R, Miles C, et al. Prediction of

558 major histocompatibility complex binding regions of protein antigens by sequence

559 pattern analysis. Proc Natl Acad Sci. 1989;86: 3296-3300.

$560 \quad$ doi:10.1073/pnas.86.9.3296

561 2. Parker KC, Bednarek MA, Coligan JE. Scheme for ranking potential HLA-A2

$562 \quad$ binding peptides based on independent binding of individual peptide side-chains.

$563 \quad$ J Immunol. 1994;152: 163-175.

564 3. Rammensee H-G, Bachmann J, Emmerich NPN, Bachor OA, Stevanović S.

565 SYFPEITHI: database for MHC ligands and peptide motifs. Immunogenetics.

$566 \quad$ 1999;50: 213-219. doi:10.1007/s002510050595 
567 4. Peters B, Bui H-H, Frankild S, Nielsen M, Lundegaard C, Kostem E, et al. A

568 Community Resource Benchmarking Predictions of Peptide Binding to MHC-I

569 Molecules. PLOS Comput Biol. 2006;2: e65. doi:10.1371/journal.pcbi.0020065

570 5. Zhao W, Sher X. Systematically benchmarking peptide-MHC binding predictors:

$571 \quad$ From synthetic to naturally processed epitopes. PLOS Comput Biol. 2018;14:

$572 \quad$ e1006457. doi:10.1371/journal.pcbi.1006457

573 6. Croft NP, Smith SA, Pickering J, Sidney J, Peters B, Faridi P, et al. Most viral

574 peptides displayed by class I MHC on infected cells are immunogenic. Proc Natl

$575 \quad$ Acad Sci. 2019;116: 3112-3117.

576 7. Moutaftsi M, Peters B, Pasquetto V, Tscharke DC, Sidney J, Bui H-H, et al. A 577 consensus epitope prediction approach identifies the breadth of murine TCD8 -

578 cell responses to vaccinia virus. Nat Biotechnol. 2006;24: 817-819.

579 8. Tscharke DC, Karupiah G, Zhou J, Palmore T, Irvine KR, Haeryfar SMM, et al. Identification of poxvirus CD8+ T cell determinants to enable rational design and characterization of smallpox vaccines. J Exp Med. 2005;201: 95-104.

9. Bui H-H, Sidney J, Peters B, Sathiamurthy M, Sinichi A, Purton K-A, et al. Automated generation and evaluation of specific MHC binding predictive tools: ARB matrix applications. Immunogenetics. 2005;57: 304-314.

587 10. O’Donnell TJ, Rubinsteyn A, Bonsack M, Riemer AB, Laserson U, Hammerbacher J. MHCflurry: Open-Source Class I MHC Binding Affinity Prediction. Cell Syst. 2018;7: 129-132.e4. doi:10.1016/j.cels.2018.05.014 
590 11. Stojanovic S. MHCLovac: MHC binding prediction based on modeled

591 physicochemical properties of peptides. [Internet]. 2019. Available:

$592 \quad$ https://pypi.org/project/mhclovac/2.0.0/

593 12. Andreatta M, Nielsen M. Gapped sequence alignment using artificial neural networks: application to the MHC class I system. Bioinformatics. 2015;32: 511517.

13. Nielsen M, Andreatta M. NetMHCpan-3.0; improved prediction of binding to MHC class I molecules integrating information from multiple receptor and peptide length datasets. Genome Med. 2016;8: 33.

600

14. Jurtz V, Paul S, Andreatta M, Marcatili P, Peters B, Nielsen M. NetMHCpan-4.0:

15. Liu I-H, Lo Y-S, Yang J-M. PAComplex: a web server to infer peptide antigen families and binding models from TCR-pMHC complexes. Nucleic Acids Res. 2011;39: W254-W260. doi:10.1093/nar/gkr434

16. Altuvia $\mathrm{Y}$, Schueler $\mathrm{O}$, Margalit $\mathrm{H}$. Ranking potential binding peptides to $\mathrm{MHC}$ molecules by a computational threading approach. J Mol Biol. 1995;249: 244250. doi:10.1006/jmbi.1995.0293

17. Singh H, Raghava G. ProPred1: prediction of promiscuous MHC Class-I binding

610 18. Reche PA, Glutting J-P, Reinherz EL. Prediction of MHC class I binding peptides using profile motifs. Hum Immunol. 2002;63: 701-709. doi:10.1016/S01988859(02)00432-9 
613 19. Peters B, Sette A. Generating quantitative models describing the sequence specificity of biological processes with the stabilized matrix method. BMC Bioinformatics. 2005;6: 132. doi:10.1186/1471-2105-6-132

616 20. Kim Y, Sidney J, Pinilla C, Sette A, Peters B. Derivation of an amino acid similarity matrix for peptide: MHC binding and its application as a Bayesian prior. BMC Bioinformatics. 2009;10: 394.

619 21. Shen Z, Reznikoff G, Dranoff G, Rock KL. Cloned dendritic cells can present exogenous antigens on both MHC class I and class II molecules. J Immunol.

22. Vita R, Overton JA, Greenbaum JA, Ponomarenko J, Clark JD, Cantrell JR, et al. The immune epitope database (IEDB) 3.0. Nucleic Acids Res. 2014;43: D405D412.

625 23. Hersperger AR, Siciliano NA, Eisenlohr LC. Comparable polyfunctionality of ectromelia virus-and vaccinia virus-specific murine T cells despite markedly different in vivo replication and pathogenicity. J Virol. 2012;86: 7298-7309.

628 24. Pedregosa F, Varoquaux G, Gramfort A, Michel V, Thirion B, Grisel O, et al. Scikit-learn: Machine learning in Python. J Mach Learn Res. 2011;12: 28252830.

631 25. Hunter JD. Matplotlib: A 2D graphics environment. Comput Sci Eng. 2007;9: 90.

632 26. Trolle T, McMurtrey CP, Sidney J, Bardet W, Osborn SC, Kaever T, et al. The Length Distribution of Class I-Restricted T Cell Epitopes Is Determined by Both Peptide Supply and MHC Allele-Specific Binding Preference. J Immunol. 2016;196: 1480-1487. doi:10.4049/jimmunol.1501721 


\section{Supporting information captions} not included. peptide lengths per allele. 
UniProt (Vaccinia virus strain Western Reserve,

https://www.uniprot.org/proteomes/UP000000344).

660

S5 Table. Overall AUCs after excluding the peptides that were identified 


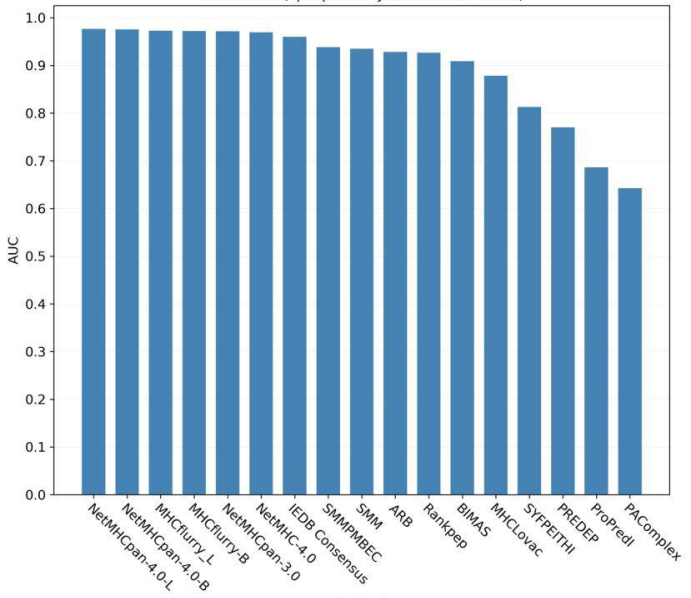




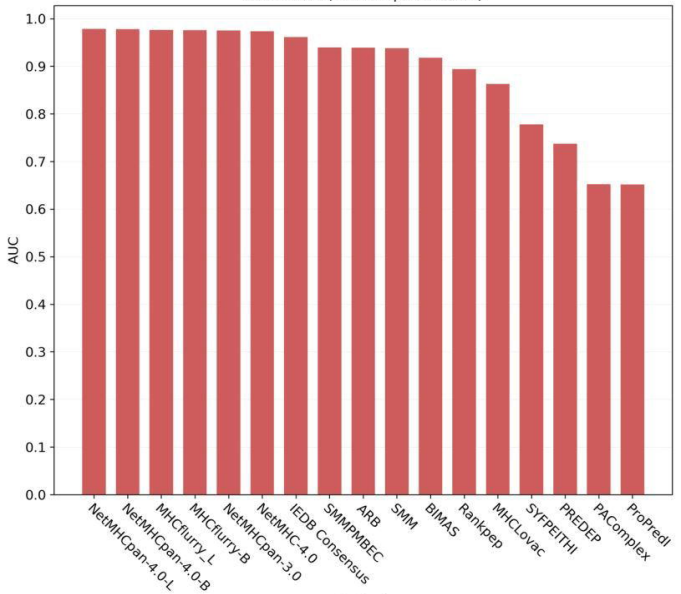




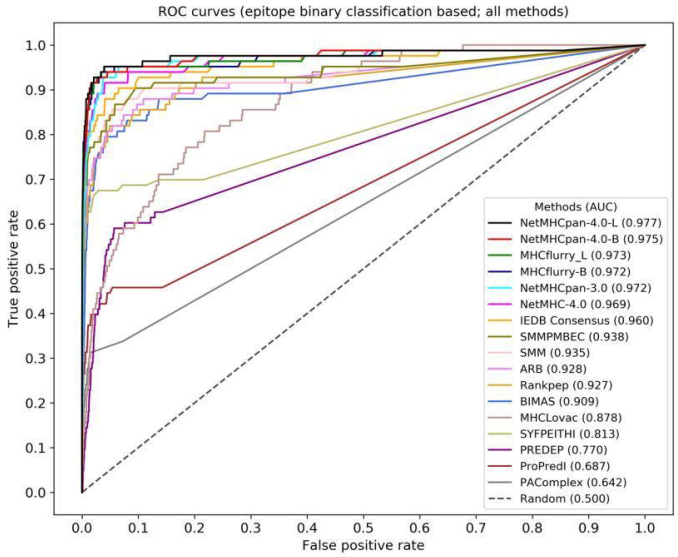




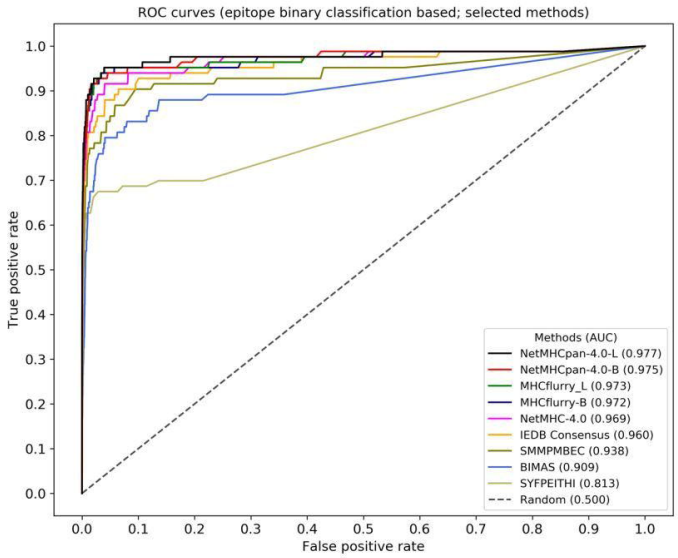


$\%$ T-cell response vs. $\%$ peptides (all methods)

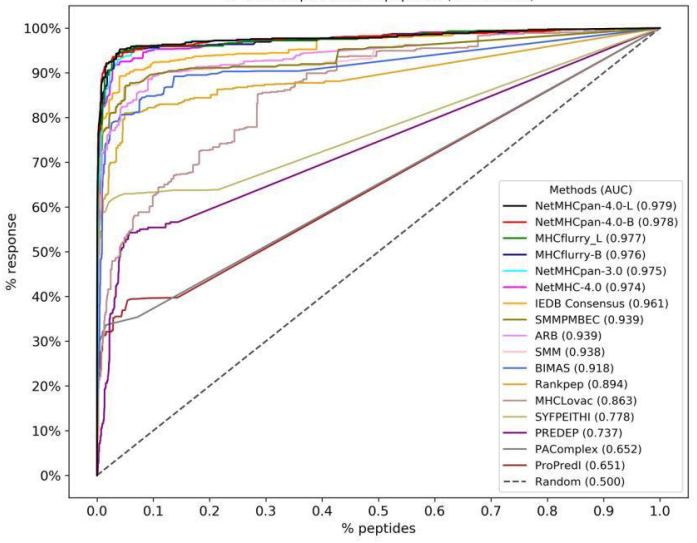


ROC curves (epitope binary classification based; all methods; zoomed-in to FPR $=0.02$ )

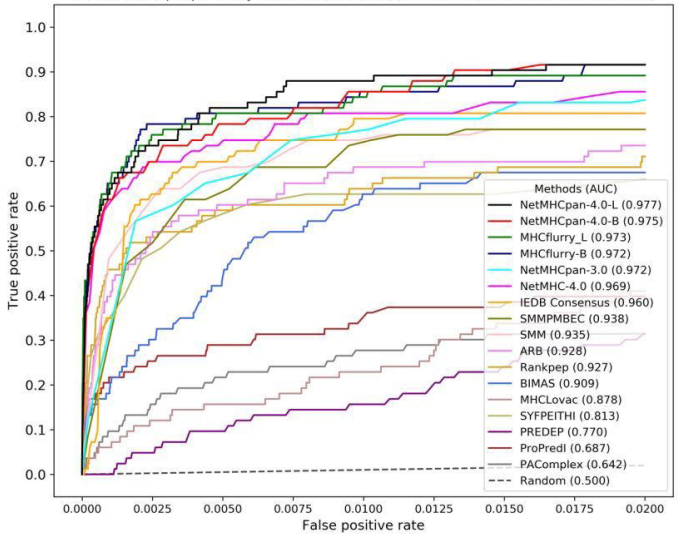


ROC curves (epitope binary classification based; selected methods; zoomed-in to FPR $=0.02$ )

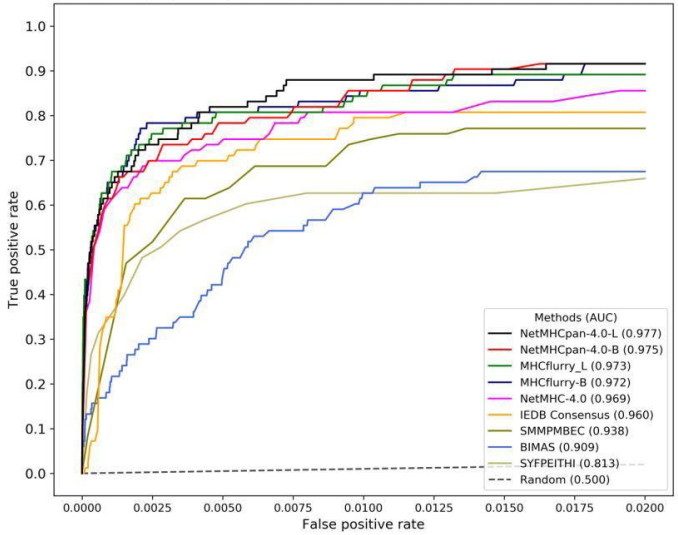


$\%$ T-cell response vs. \% peptides (all methods; zoomed-in to FPR $=0.02$ )

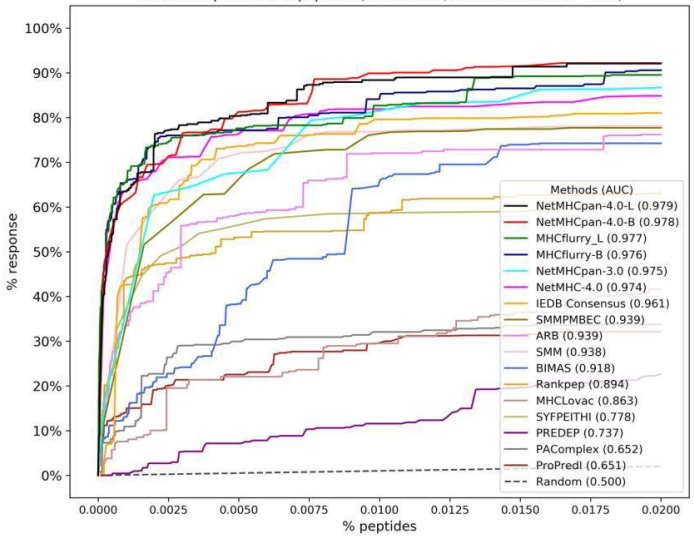


$\%$ peptides needed to capture $50 \%$ epitopes

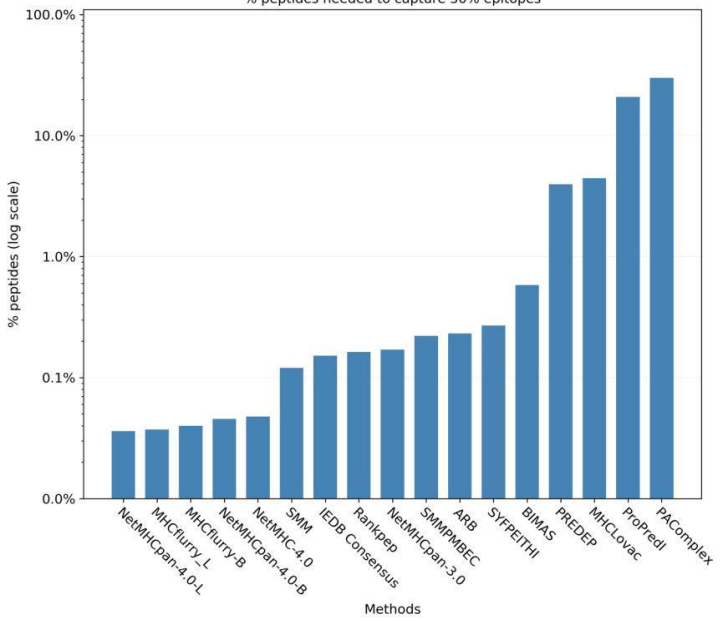


$\%$ peptides needed to capture $50 \%$ response

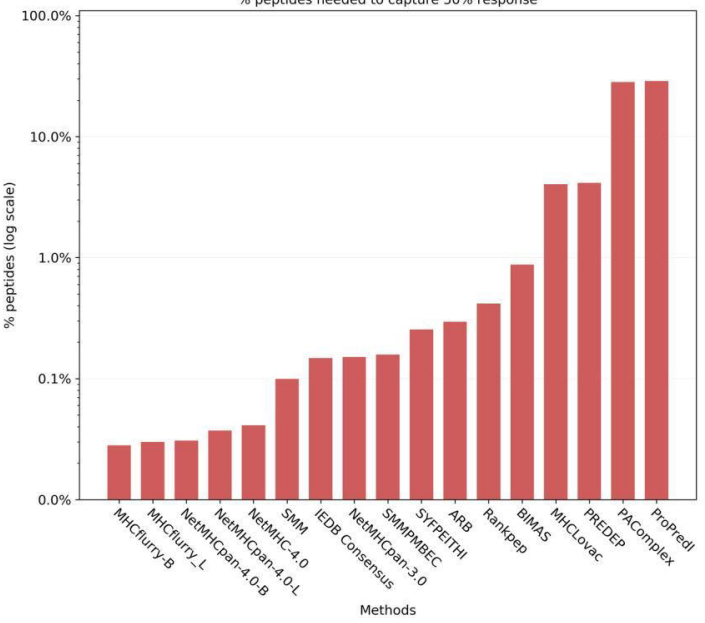


$\%$ peptides needed to capture $75 \%$ epitopes

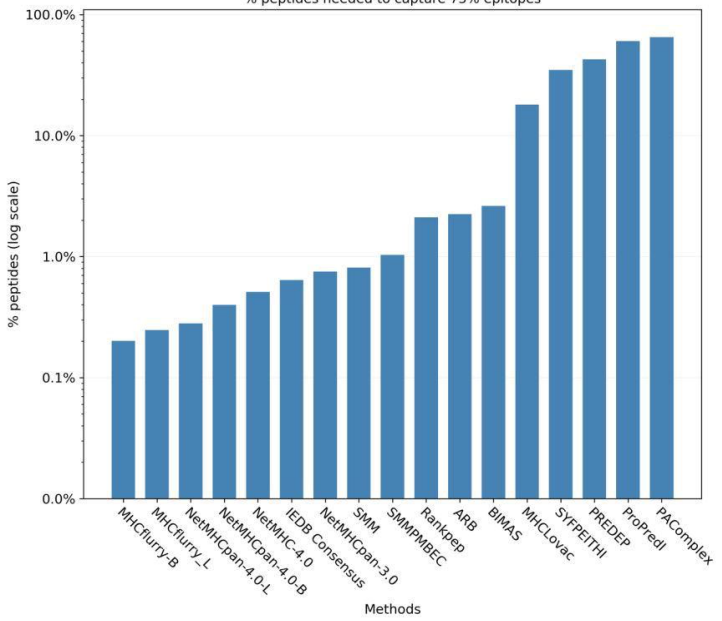


$\%$ peptides needed to capture $75 \%$ response

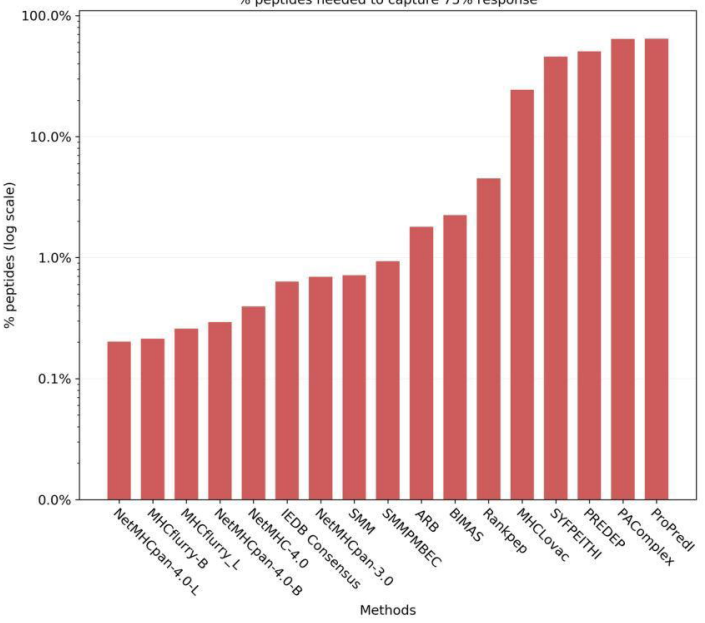




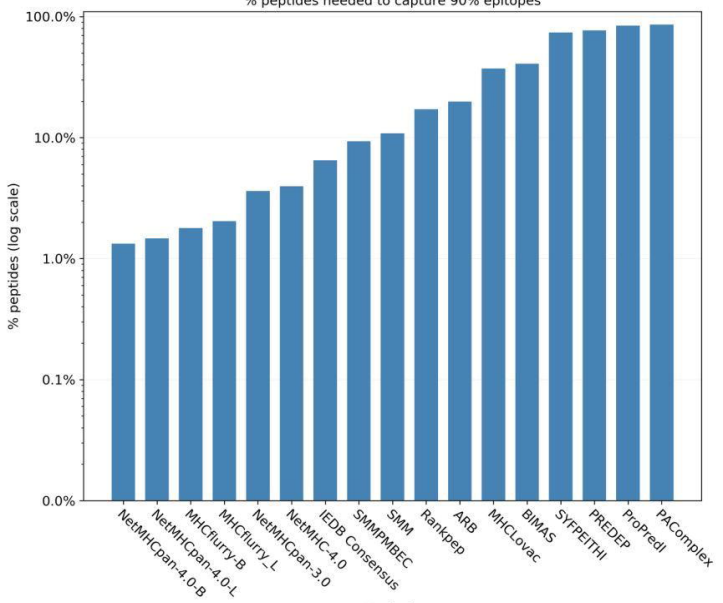


$\%$ peptides needed to capture $90 \%$ response

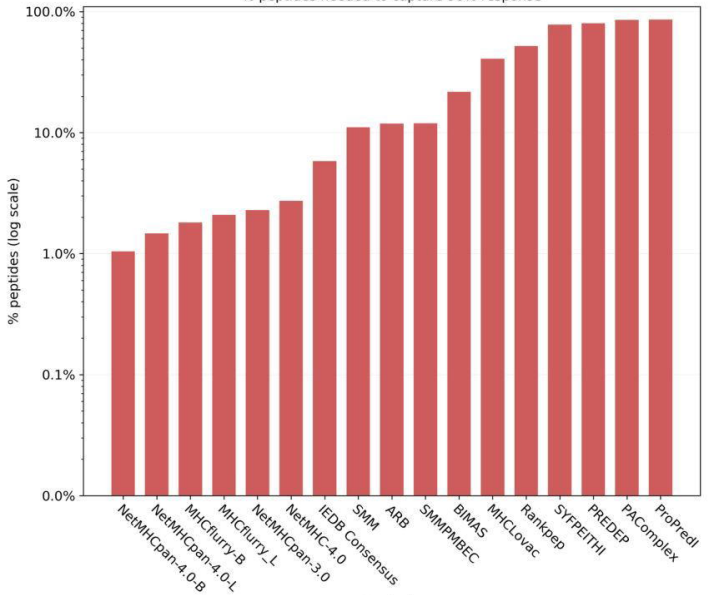

\section{Methods}


$\%$ epitopes captured in top 172 peptides

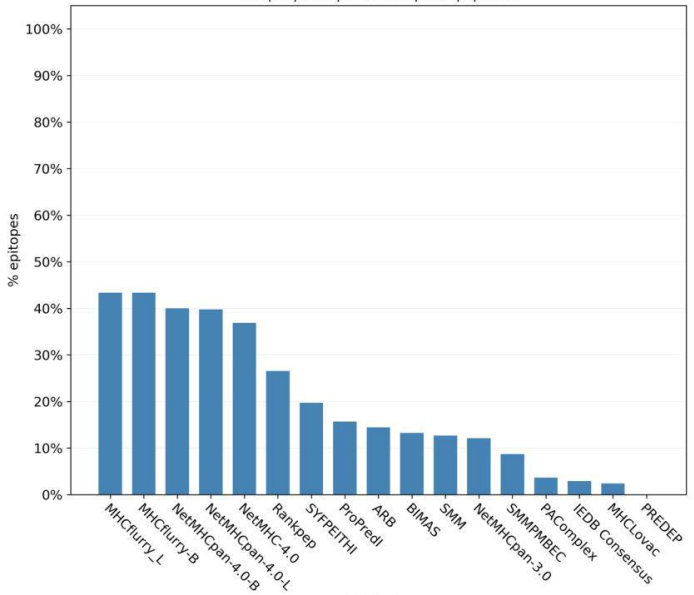

Methods 
$\%$ T-cell response captured in top 172 peptides

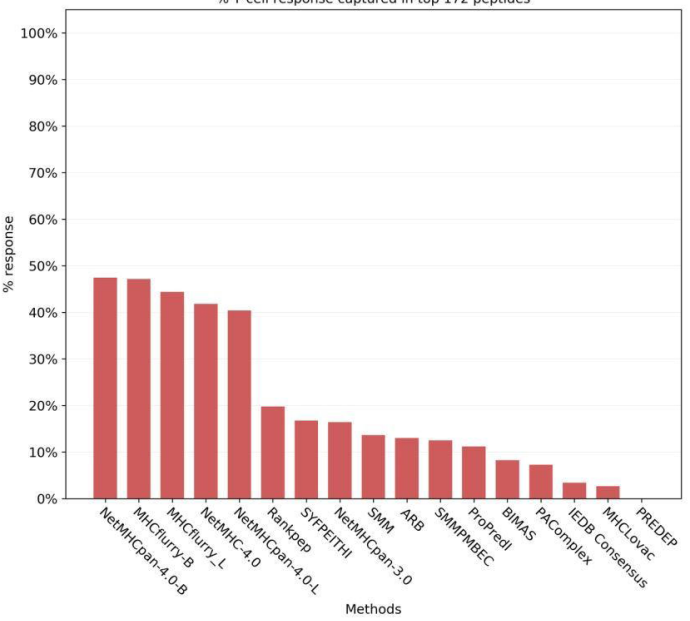




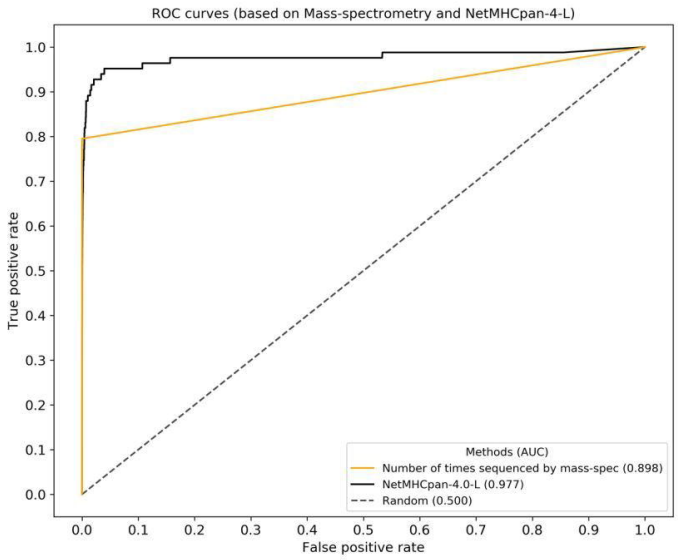


ROC curves (based on Mass-spectrometry and NetMHCpan-4-L; zoomed-in to FPR $=0.02$ )

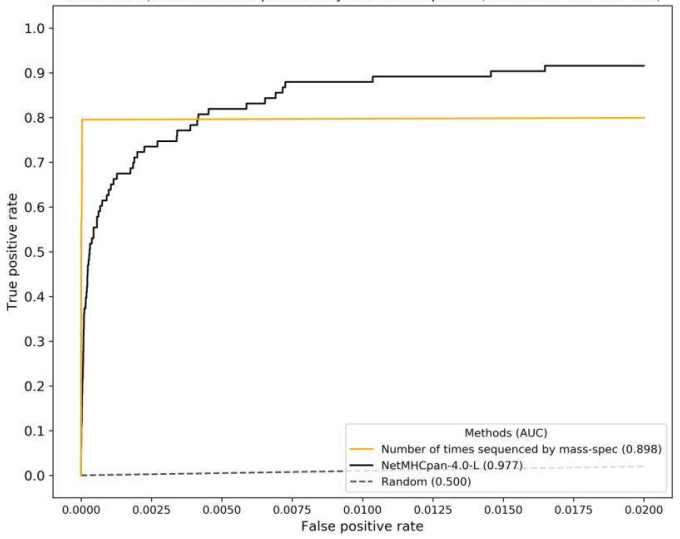


Average time in seconds to do prediction for a 1000 residue sequence

A. Comparing methods

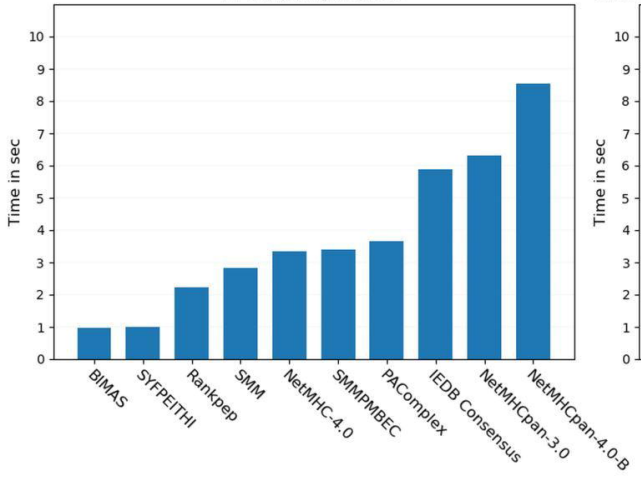

B. Comparing method types

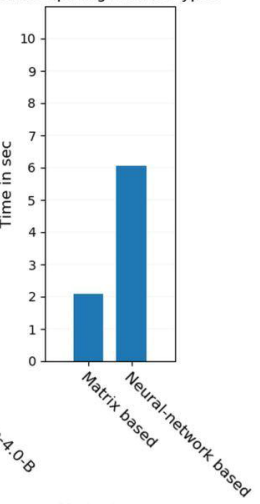

Method types 